\title{
Insulina en poblaciones especiales: resistencia a la insulina, obesidad, embarazo, adultos mayores y enfermedad renal crónica
}

\section{Insulin in special populations: Insulin resistance, obesity, pregnancy, older adults, and chronic kidney disease}

\author{
DANiel Elías-López ${ }^{1 *}$ y Aldo FerReira-HeRMosilio² \\ 'Unidad de Investigación de Enfermedades Metabólicas, Departamento de Endocrinología y Metabolismo, Instituto Nacional de \\ Ciencias Médicas y Nutrición Salvador Zubirán; ${ }^{2}$ Unidad de Investigación Médica en Enfermedades Endocrinas, Centro Médico \\ Nacional Siglo XXI, Instituto Mexicano del Seguro Social. Ciudad de México, México
}

\section{RESUMEN}

A 100 años del descubrimiento de la insulina, uno de los avances científicos en medicina más importantes del siglo pasado, un gran número de personas que viven con diabetes han incrementado su supervivencia y mejorado su calidad de vida. Ahora que vemos los enormes esfuerzos científicos para desarrollar las vacunas contra el nuevo coronavirus tipo 2 causante del síndrome respiratorio agudo severo (SARS-CoV-2), podemos entender que los esfuerzos coordinados siempre llevan a un beneficio colectivo, como ocurrió hace justo 100 años y como ha ocurrido a lo largo del desarrollo de la medicina. Aparte de su amplia utilización en pacientes con diabetes, la insulina se usa en escenarios en donde frecuentemente no existe tanta evidencia científica proveniente de grandes ensayos clínicos. Entre estas condiciones podemos citar la resistencia extrema a la insulina, la obesidad extrema, el embarazo, la enfermedad renal crónica y los pacientes adultos mayores. El tratamiento con insulina en estas condiciones presenta ciertas consideraciones y peculiaridades: uso de dosis mayores, mayor riesgo de eventos de hipoglucemia, presentaciones con una mayor concentración y monitoreo de glucosa más frecuente, entre otros. La presente revisión tiene como objetivo explorar, analizar y discutir la evidencia más reciente de estos temas.

Palabras clave: Diabetes. Insulina y embarazo. Resistencia extrema a la insulina. Obesidad extrema. Enfermedad renal crónica. Diabetes en adulto mayor.

\begin{abstract}
100 years after the discovery of insulin, one of the most important scientific advances in medicine in the last century, a large number of people living with diabetes have increased their survival and improved their quality of life. Now that we realize the enormous scientific efforts to develop vaccines against the new type 2 coronavirus causing severe acute respiratory syndrome (SARS-CoV-2), we can understand that coordinated efforts always lead to a collective benefit, as happened just 100 years ago and as has happened throughout the development of medicine. Apart from its widespread use in patients with diabetes, insulin is used in settings where there is often less scientific evidence from large clinical trials. These conditions include extreme insulin resistance, extreme obesity, pregnancy, chronic kidney disease (CKD), and elderly patients. Insulin treatment in these conditions presents certain considerations and peculiarities: use of higher doses; higher risk of hypoglycemia events; higher concentration presentations; more frequent glucose monitoring; among others. The present review aims to explore, analyze and discuss the most recent evidence on these issues.
\end{abstract}

Key words: Diabetes. Insulin and pregnancy. Extreme insulin resistance. Extreme obesity. Chronic kidney disease. Diabetes in the elderly.
Correspondencia:

*Daniel Elías-López

E-mail: drdanielelias@outlook.com
Fecha de recepción: 09-08-2021

Fecha de aceptación: 27-09-2021

DOI: 10.24875/RME.M21000013
Disponible en internet: 05-11-2021 RevMexEndocrinol Metab Nutr.2021;8:(SUPL.3):59-71

2462-4144 / ๑ 2021 Sociedad Mexicana de Nutrición y Endocrinología, AC. Publicado por Permanyer. Este es un artículo open access bajo la licencia CC BY-NC-ND (http://creativecommons.org/licenses/by-nc-nd/4.0/). 


\section{RESISTENCIA EXTREMA A LA INSULINA}

El tratamiento con insulina en las personas que viven con diabetes tiene un lugar muy importante dentro de las guías de tratamiento ${ }^{1-4}$. La insulina tiene diversas acciones metabólicas que ayudan a mantener la homeostasis de la glucosa. Entre estas, se han descrito: promueve la utilización de la glucosa en los órganos periféricos, permite suprimir la producción hepática de glucosa, así como la lipólisis en el tejido adiposo. Sin embargo, además de sus efectos en estos órganos «clásicos», también tienen efectos en otros, como el cerebro, las células beta pancreáticas, el corazón, el ovario y el endotelio vascular, entre otros ${ }^{5}$.

La insulina ejerce sus efectos biológicos cuando se une al receptor de la insulina (INSR), el cual es una glucoproteína heterotetramérica que se encuentra en la superficie celular6. Las alteraciones que ocurren en cualquier punto de la cascada de la vía de señalización de la insulina podrían contribuir a la patogénesis de la resistencia a la insulina (RI), incluyendo los casos de resistencia extrema. Cuando hablamos de RI se engloba a la menor capacidad de respuesta a la eliminación de la glucosa mediada por la insulina, así como a una menor capacidad de inhibición de la producción hepática de glucosa y de la lipólisis del tejido adiposo. Una de las respuestas compensatorias que ocurren en las personas con RI es la hiperinsulinemia. Debido a que la insulina tiene varias funciones, la hiperinsulinemia compensatoria suele tener resultados fisiopatológicos adversos en otros órganos y sistemas ${ }^{7}$.

El término de RI fue utilizado por primera vez varios años después de la introducción de la terapia con insulina en 1922, para describir a aquellos pacientes con diabetes que requerían dosis cada vez mayores de insulina para controlar la hiperglucemia. La mayoría de estos pacientes desarrollaron una RI secundaria a los anticuerpos dirigidos contra la insulina terapéutica, que en aquella época era impura y derivada de especies no humanas ${ }^{8}$. Los anticuerpos antiinsulina en títulos suficientes para alterar la acción de la insulina son extremadamente raros en pacientes actuales tratados con insulina humana recombinante.
El espectro de trastornos clínicos en los que la RI desempeña un papel importante ha cambiado notablemente en los últimos años y es muy amplio. La RI está estrechamente asociada a importantes problemas de salud pública, como la obesidad, la diabetes mellitus tipo 2 (DM2), la hipertensión arterial, la enfermedad coronaria, las dislipidemias y a otras entidades cardiometabólicas ${ }^{9}$. Bajo este espectro de alteraciones, la epidemia mundial de obesidad juega un papel pivote. La RI constituye, por tanto, una condición fisiopatológica subyacente para muchos trastornos clínicos, normalmente agrupados bajo el término «síndrome metabólico». Por otro lado, los síndromes de resistencia extrema a la insulina son raros, y aunque su prevalencia no ha sido descrita, puede constituir entre el $0.1-0.5 \%$ de las personas que se atienden en clínicas especializadas en diabetes $^{10}$. La resistencia extrema a la insulina se presenta como una respuesta muy baja a los efectos biológicos de la insulina, con la hiperinsulinemia consecuente y alteraciones en la respuesta de la glucosa a la insulina endógena y exógena.

\section{Características clínicas}

Aunque la principal causa de RI en la actualidad es la obesidad, hay otras causas descritas, como se observa en la tabla 1.

Algunas de las primeras características clínicas clásicas de la hiperinsulinemia crónica por Rl aparecen en la piel, como la acantosis nigricans y los acrocordones; sin embargo, las manifestaciones clínicas suelen ser variables. La fisiopatología exacta de estas alteraciones en la piel no se conoce con cabalidad, pero la evidencia sugiere que los niveles altos de insulina reaccionan de forma cruzada con el receptor del factor de crecimiento insulínico tipo 1 en queratinocitos y fibroblastos de la dermis ${ }^{11}$. En las mujeres, las manifestaciones iniciales pueden estar relacionadas con el hiperandrogenismo (asociada al hiperinsulinismo compensatorio) como el hirsutismo, ovarios poliquísticos, irregularidades menstruales u oligomenorrea. Otra característica frecuentemente observada en estados de Rl severa es la alteración de la homeostasis de la glucosa. El deterioro de la tolerancia a la glucosa y la DM se desarrollan cuando la 
Tabla 1. Principales causas de resistencia a la insulina

- Condiciones hereditarias relacionadas con la resistencia a la insulina

- Leprechaunismo (mutaciones del receptor de insulina)

- Síndrome de Rabson-Mendenhall (mutaciones del receptor de insulina)

- Síndrome de resistencia a la insulina tipo A (mutaciones del receptor de insulina en algunos casos, defecto desconocido de señalización en la mayoría)

- Algunas lipodistrofias

- Resistencia secundaria a la insulina

- Obesidad (los ácidos grasos libres y las adipocitocinas pueden contribuir)

- Estrés, infecciones debidas al exceso de hormonas contrarreguladoras (cortisol, catecolaminas, hormona del crecimiento, glucagón)

- Medicamentos (p. ej., glucocorticoides, antirretrovirales para el virus de inmunodeficiencia humana, anticonceptivos orales)

- Sedentarismo

- Embarazo (lactógeno placentario)

- Mediada por inmunidad (anticuerpos antiinsulina, anticuerpos antirreceptor de insulina en el síndrome tipo B)

- Varios (inanición, uremia, cirrosis, cetoacidosis)

- Consecuencias de la resistencia a la insulina

- La mayoría de los casos de diabetes mellitus tipo 2

- Enfermedades cardiovasculares, hipertensión

- Síndrome de ovario poliquístico

- Síndrome metabólico

- Cáncer relacionado a la obesidad (varios)

respuesta compensatoria de las células beta a la RI es insuficiente para regular el metabolismo de la glu$\operatorname{cosa}^{12}$. Esto puede reducir la utilidad diagnóstica de las mediciones de insulina. Otras manifestaciones incluyen a la dislipidemia (específicamente la hipertrigliceridemia), enfermedad del hígado graso no alcohólico, pérdida de tejido adiposo, musculatura anormal, rasgos acromegaloides y otros trastornos del crecimiento $^{10}$.

Existen distintos métodos disponibles para estimar la sensibilidad/resistencia a la insulina. Estos métodos van desde procedimientos invasivos, complejos, que requieren mucho tiempo y trabajo y que habitualmente son utilizados con fines de investigación, hasta pruebas sencillas que implican una única muestra de sangre en ayunas. Es importante comprender los conceptos fisiológicos en los que se basa cada método para que los méritos y las limitaciones relativas de cada uno de ellos se correspondan con las aplicaciones propuestas y los datos se interpreten correctamente. También conviene identificar que el laboratorio donde realizaremos el estudio tenga los estándares de calidad necesarios. El método del clamp de glucosa es el estándar de referencia para la medición directa de la sensibilidad a la insulina. En cuanto a los sustitutos simples, el Quantitative Insulin Sensitivity Check Index (QUICKI) y el Homeostasis Model Assessment (HOMA) se encuentran entre los mejores y más ampliamente validados ${ }^{13}$.

Los niveles de insulina no se evalúan frecuentemente en la práctica clínica, es por ello que muchos de los síndromes de resistencia extrema a la insulina están infradiagnosticados. En ayunas, niveles de insulina superiores a $50-70 \mu \mathrm{U} / \mathrm{mL}$ o niveles que superan los $350 \mu \mathrm{U} / \mathrm{mL}$ tras una prueba de tolerancia oral a la glucosa pueden indicar que estamos ante una Rl extrema ${ }^{10}$. Sin embargo, no existen criterios bioquímicos de diagnóstico clínico universalmente aceptados de resistencia extrema a la insulina.

Al evaluar fenotipos graves de $\mathrm{Rl}$, se debe medir e interpretar la insulina y el péptido $C$ en ayunas (en el contexto de los niveles de glucosa circulante). La hiperinsulinemia extrema debe sugerir estudios adicionales para detectar insulina degradada en el tejido subcutáneo, la presencia de mutaciones de la insulina o del receptor de insulina, la presencia de anticuerpos circulantes anti-INSR, y niveles de leptina en pacientes con datos clínicos de lipodistrofia completa y posible deficiencia de leptina. 


\section{Acciones terapéuticas}

Las acciones terapéuticas de las personas con resistencia extrema a la insulina tienen como objetivo revertir o atenuar la RI. Se deben incluir modificaciones profundas del estilo de vida centradas en la reducción de calorías, disminución del peso y aumento de la actividad física, medicamentos hipoglucemiantes orales e inyectables, insulina e hipolipemiantes.

Característicamente los pacientes con RI (sobre todo aquellos con resistencia extrema) requieren grandes cantidades de insulina exógena para mantener los niveles de glucosa adecuados. La resistencia severa a la insulina ha sido definida como la necesidad de dosis de insulina que supera las $2 \mathrm{U} / \mathrm{kg} /$ día o las 200 U/día durante más de dos días ${ }^{14}$. Con volúmenes tan grandes de insulina, se puede causar una molestia considerable en el lugar de la inyección, así como un aumento en la agregación de hexámeros y una mayor duración del efecto (considere $2 \mathrm{~mL}$ para una dosis de 200 unidades de insulina U100) ${ }^{14}$.

La concentración de las insulinas tradicionales es de 100 unidades $/ \mathrm{mL}$ (U100). Desde hace varios años se han realizado distintas preparaciones que han permitido la concentración de la insulina en un volumen menor, como la insulina degludec 200 unidades/mL (U200), lispro 200 unidades/mL (U200) y glargina 300 unidades $/ \mathrm{mL}$ (U300), así como la insulina regular U500 que contiene $500 \mathrm{U} / \mathrm{mL}$ y que fue la primera insulina concentrada en desarrollarse ${ }^{14}$.

La insulina U200 lispro está aprobada en EE.UU. por la Food and Drug Administration (FDA) para mejorar el control glucémico de los pacientes con diabetes de tipo 1 y 2 , en particular, en pacientes que requieren altas dosis de insulina antes de las comidas y que se benefician de menores volúmenes, así como la utilización de menos plumas ${ }^{15}$. Esta pluma no se encuentra disponible en México actualmente.

La insulina degludec U200 tiene la misma vida media que la insulina degludec U100, y se alcanza un estado estable dentro de los primeros tres días de su administración. Esta insulina podría considerarse para los pacientes que requieren dosis elevadas de insulina basal, por ejemplo los pacientes con resistencia extrema a la insulina, ya que pueden administrarse dosis de hasta $160 \mathrm{U}$ en una sola inyección.
El estudio BEGIN Low-Volume ${ }^{16}$ comparó insulina degludec U200 vs. glargina U100 en pacientes vírgenes a insulina, en descontrol glucémico por hipoglucemiantes orales. Al final del estudio se demostró la misma eficacia entre ambos, sin diferencias significativas en el peso ni en las unidades de insulina administradas. Se identificaron menores episodios de hipoglucemia en el grupo de degludec U200 (20.5\%) vs. glargina U100 (30.7\%), sin diferencias en las tasas de eventos de hipoglucemias graves. Además, no hubo diferencias en la dosis de insulina ni en el cambio de peso durante el periodo de estudio entre los grupos de tratamiento. Esta insulina tampoco se encuentra disponible actualmente en nuestro país.

La insulina glargina U300, que se encuentra disponible en nuestro país desde hace algunos años, es una insulina basal concentrada que contiene 300 unida$\mathrm{des} / \mathrm{mL}$. Esta forma más concentrada de glargina U300 retrasa la redisolución del depósito subcutáneo después de la inyección, lo que le permite una farmacocinética más plana y prolongada comparada con glargina U100, pero es ligeramente menos potente cuando se compara unidad por unidad ${ }^{17}$. Los análisis de sus estudios principales (EDITION 1-3) muestran una eficacia comparable entre glargina U300 y U100, con tasas de hipoglucemia confirmada o grave menores con glargina U300 que con glargina U100 durante la noche y en cualquier momento. Los eventos adversos y tolerabilidad fueron comparables entre ambos tratamientos ${ }^{18}$.

La insulina U500 es cinco veces más concentrada y contiene 500 unidades de insulina por $\mathrm{mL}$. Esto permite la inyección de grandes dosis de insulina en la quinta parte del volumen de la insulina U100. La insulina U500 fue aprobada por la FDA en 1994, para pacientes resistentes a la insulina con DM2 que necesitan más de 200 unidades de insulina al día ${ }^{19}$. La insulina U500 tiene acciones tanto prandiales como basales, y puede inyectarse como monoterapia cada dos días 20 .

En los pacientes con resistencia extrema a la insulina, además de la insulina, se recomienda el uso concomitante de terapias que ayuden a mejorar la RI. Existen datos acerca del uso de la metformina y las tiazolidinedionas (TZD) para mejorar la tolerancia a 
la glucosa, en parte por el aumento de la sensibilidad a la insulina. La metformina se considera la farmacoterapia de primera línea para mejorar la sensibilidad a la insulina en pacientes con lipodistrofias ${ }^{21}$. Además, las TZD también aumentan los niveles de adiponectina, por lo que actualmente se apoya el uso de las TZD, especialmente la pioglitazona, para mejorar el perfil metabólico en pacientes con lipodistrofia parcial ${ }^{16}$. Aunque no se han estudiado ampliamente, las terapias que mejoran el efecto incretina, como los agonistas del receptor del péptido similar al glucagón 1 (AR-GLP-1), así como los inhibidores del transportador de glucosa de sodioglucosa 2 podrían utilizarse en la lipodistrofia ${ }^{22}$.

\section{USO DE INSULINA EN OBESIDAD EXTREMA}

La obesidad sigue siendo un problema mundial de salud muy importante. En la actualidad, se estima que la obesidad afecta a uno de cada tres adultos en el mundo ${ }^{23}$. México no ha escapado a esta epidemia, y actualmente la obesidad es considerada uno de los principales problemas de salud pública en el país, ya que las comorbilidades asociadas a su presencia constituyen las principales causas de morbimortalidad entre su población, como diabetes, hipertensión arterial y enfermedad cardiovascu$\operatorname{lar}^{24}$. Los datos de la Encuesta Nacional de Salud y Nutrición (ENSANUT) 2018-2019 en México indican que la prevalencia de obesidad sigue aumentando en nuestra población. A nivel nacional el $75.2 \%$ de los adultos tienen sobrepeso (39.1\%) u obesidad $(36.1)^{25}$.

\section{Epidemiología de la obesidad extrema en México}

La prevalencia de obesidad extrema ha tenido una tendencia al alza en distintas partes del mundo, y también en México. Los datos de la ENSANUT 20182019 informan que en el periodo 2000-2018 la mayor carga de obesidad se observó a mayor grado de obesidad, la cual tuvo un incremento del $28.8 \%$ para obesidad grado I, mientras que para la obesidad grado III o extrema el aumento fue del $96.5 \%$ en el mismo periodo. En todos los años, la prevalencia de obesidad mórbida fue mayor en mujeres que en hombres ${ }^{24}$.

\section{Retos clínicos del uso de insulina en obesidad extrema}

Uno de los varios problemas asociados a la mayor prevalencia de obesidad extrema es la falta de infraestructura y capacidades específicas para su atención. Otro reto al que nos enfrentamos los clínicos tiene que ver más con su tratamiento, ya cuando los pacientes con obesidad extrema desarrollan comorbilidades como diabetes, hipertensión o dislipidemias, frecuentemente necesitan un tratamiento más intensivo, incluyendo dosis mayores a las habituales. A mayor grado de obesidad, puede haber mayor grado de $\mathrm{Rl}$, lo que contribuye a mayores requerimientos de insulina para lograr metas de control entre los pacientes con obesidad y diabetes ${ }^{26}$. Esto implica para dichos pacientes un mayor aumento de peso, mayor riesgo de eventos de hipoglucemia, un esquema de insulinización más complejo y mayor gasto económico debido a las dosis más altas.

Como se ha mencionado en el tema de resistencia extrema a la insulina, los pacientes con diabetes que requieren más de 1 unidad $/ \mathrm{kg} /$ día se consideran con $\mathrm{Rl}$, y los que necesitan más de 2 unidades/ $\mathrm{kg} /$ día tienen una resistencia grave. Además, una dosis total de insulina/día > 200 unidades se considera también como una resistencia grave ${ }^{14}$ a la insulina. Estas características relacionadas con el tratamiento con insulina son más frecuentes entre aquellos pacientes con obesidad extrema, y si consideramos el hecho de que la obesidad extrema sigue y seguirá aumentando de forma alarmante, tendremos que estar preparados para enfrentar este reto de manera adecuada.

Las dosis altas de insulina crean algunos problemas prácticos en los pacientes y los clínicos, por ejemplo el tener que inyectar un gran volumen de insulina (con la concentración de insulina habitual U-100) y alteraciones en la farmacocinética de la insulina (en el inicio y la duración de la actividad de la insulina) 
Tabla 2. Inicio, pico y duración de las insulinas concentradas

\begin{tabular}{|c|c|c|c|c|}
\hline Tipo de insulina & Inicio de acción & Pico & Duración & $\begin{array}{c}\text { Disponibilidad } \\
\text { en México }\end{array}$ \\
\hline U200 Lispro (Humalog ${ }^{\circledR}$ U200) & 15 minutos & 30 a 90 minutos & 4 a 5 horas & No \\
\hline U200 (Tresiba $\left.{ }^{\oplus}\right)$ & 1 a 4 horas & Sin picos significativos & $\sim 42$ horas & No \\
\hline U300 Glargina (Toujeo ${ }^{\oplus}$ ) & 6 horas & Sin picos significativos & 24-36 horas & Sí \\
\hline U500 Insulina regular (Humulin R U500 ${ }^{\circledR}$ ) & 30 minutos $a>1$ hora & 2 a 4 horas & $\sim 21$ horas & No \\
\hline
\end{tabular}

Adaptada de Umpierrez, et al., $2020^{14}$.

debido al mayor volumen (más de $1 \mathrm{~mL}$ ). Por otro lado, las jeringas de insulina disponibles pueden suministrar un máximo de 100 unidades, y los dispositivos (plumas) solo pueden suministrar entre 60-80 unidades por inyección. Por lo tanto, aquellos pacientes con obesidad extrema y resistencia grave a la insulina necesitarán varias inyecciones al día para lograr la dosis prescrita, lo que se reflejaría también en un mayor gasto. Esto ha generado el desarrollo de estrategias como concentrar la insulina, lo cual permite administrar mayores unidades de insulina en un menor volumen, lo que disminuye el dolor y el número de inyecciones al día ${ }^{26}$. Para cualquier paciente con obesidad y diabetes, incluyendo aquellos con obesidad extrema, las guías de tratamiento recomiendan el uso concomitante de otras terapias que no tengan impacto en el peso, incluso que promuevan la pérdida de peso, así como sensibilizadores de insulina y fármacos con menor riesgo de hipoglucemia ${ }^{1-4}$.

\section{Falta de opciones de insulinas concentradas en México}

En el tema previo de RI se hace referencia a las insulinas concentradas que se encuentran en otros países, así como los principales datos que confirman su eficacia y seguridad en los distintos estudios clínicos, que se resumen en la tabla 2 . En la actualidad, en México solo está disponible una insulina concentrada, la insulina glargina U-300, la cual ha demostrado en su programa científico EDITION datos de eficacia y seguridad, con beneficios adicionales sobre incidencia y eventos de hipoglucemia. Aunque en la práctica clínica diaria de nuestro país no es frecuente el uso de grandes cantidades de insulina, sería deseable disponer de un mayor número de insulinas concentradas, que sean accesibles y asequibles para nuestra población.

\section{USO DE INSULINA EN EL EMBARAZO}

\section{Consecuencias de la hiperglucemia en el embarazo}

En los últimos años, se ha prestado mayor atención a la mejora de la atención preconcepcional, concepcional y posparto en las mujeres en edad reproductiva. Distintos estudios han informado respecto a la mejoría en los desenlaces obstétricos y perinatales cuando hay un adecuado control glucémico en las pacientes con diabetes pregestacional y gestacional ${ }^{27}$. Antes de la disponibilidad de la insulina, las mujeres con diabetes difícilmente lograban llegar a la edad fértil, tener un embarazo y menos lograr un adecuado desarrollo del feto a lo largo de la gestación ${ }^{28}$. El advenimiento de la insulina hace 100 años permitió superar estos obstáculos; sin embargo, ahora nos enfrentamos a otros retos no menos importantes, el disminuir la morbimortalidad fetal y materna debido a la diabetes preexistente o que se desarrolla durante el embarazo. Entre las pacientes con diabetes pregestacional, el énfasis en la importancia del control estricto de la glucemia, la administración de suplementos de ácido fólico, la interrupción de medicamentos potencialmente perjudiciales, el fomento de la pérdida de peso en las mujeres con sobrepeso u obesidad y la optimización de las afecciones médicas asociadas, incluidas las complicaciones de la diabetes, son componentes importantes de la atención preconcepcional. Los niveles elevados 
de glucosa, meses previos a la concepción y a lo largo del primer trimestre, confieren un riesgo significativo dependiente de la dosis de anomalías congénitas, incluyendo defectos cardiacos y esqueléticos, así como de aborto ${ }^{29}$. En caso de diabetes mellitus gestacional, el descontrol glucémico se asocia a consecuencias maternas y fetales, como mayor riesgo de preeclampsia y macrosomía, por lo que el manejo de la hiperglucemia, la monitorización del embarazo, el manejo de las complicaciones del embarazo, la atención posparto y el seguimiento a largo plazo son fundamentales (Tabla 3).

En las últimas décadas, y con el advenimiento de la pandemia mundial de sobrepeso y obesidad materna, se han elevado las tasas de diabetes gestacional y pregestacional. En el último reporte del Atlas de diabetes 2019 de la Federación Internacional de Diabetes (IDF) se informa que la hiperglucemia durante el embarazo afecta a uno de cada seis embarazos en el mundo, por lo tanto la diabetes en el embarazo es actualmente una preocupación mundial de primer orden, con implicaciones tanto para la madre como para la descendencia ${ }^{30}$.

\section{Tratamiento con insulina durante el embarazo}

Para evitar complicaciones fetales y maternas, se recomienda alcanzar adecuados objetivos glucémicos antes y durante el embarazo ${ }^{31}$, con objetivos de glucosa en ayunas $<95 \mathrm{mg} / \mathrm{dl}$ y posprandiales $<140 \mathrm{mg} / \mathrm{dl}$ y $<120 \mathrm{mg} / \mathrm{dl}$ a la hora y 2 horas respectivamente. Así mismo, el objetivo de hemoglobina glucosilada ( $\mathrm{HbA} 1 \mathrm{c})$ es $<6.5 \%$ antes de la concepción y $<6 \%$ durante el embarazo, idealmente bajo un esquema terapéutico con bajo riesgo de hipoglucemias.

Debido su mayor eficacia en la reducción de la glucosa y su incapacidad para atravesar la placenta, la insulina es el agente recomendado para el tratamiento de la hiperglucemia en el embarazo cuando las intervenciones en el estilo de vida o el tratamiento oral no consiguen garantizar la consecución de los objetivos glucémicos. Debido a la falta de datos de seguridad a largo plazo sobre el uso de metformina en el embarazo, la Asociación Americana de
Tabla 3. Riesgo de anomalías congénitas según los valores de hemoglobina glucosilada (HbA1c) antes del embarazo o durante el primer trimestre del embarazo

\begin{tabular}{lc}
\hline HbA1c (\%) & $\begin{array}{c}\text { Riesgo absoluto de } \\
\text { anomalía congénita (\%) }\end{array}$ \\
\hline$<5.6$ & 1.6 \\
\hline $5.7-6.5$ & $2.2-3$ \\
\hline $6.6-7.8$ & $3-3.9$ \\
\hline $7.9-9.1$ & $3.9-7.5$ \\
\hline$>9.1$ & $1.7-20$ \\
\hline
\end{tabular}

Adaptada de Buschur, et al., $2021^{27}$.

Diabetes (ADA) y el Colegio Americano de Obstetras y Ginecólogos (ACOG) recomiendan la insulina como agente de primera línea para el tratamiento de la diabetes en el embarazo, incluyendo a la diabetes preexistente y la diabetes mellitus gestacional ${ }^{11,32}$.

Distintas insulinas basales y de corta duración son seguras durante el embarazo. Las insulinas humanas disponibles no atraviesan la placenta, a menos que estén unidas a un anticuerpo. Aunque hay menos datos de seguridad sobre el uso de análogos de insulina basal en el embarazo, parecen también ser seguras. La insulina detemir y la NPH (Neutral Protamine Hagedorn) se prefieren dado sus datos de seguridad. La insulina glargina ha demostrado tener una mayor afinidad con el receptor de la insulina, que participa en la implantación del embrión en el útero, lo que podría contribuir a efectos perjudiciales en el embarazo, como el aborto, el sobrecrecimiento fetal y malformaciones congénitas. Sin embargo, en un metaanálisis publicado hace algunos años no se demostró ninguna diferencia en los resultados maternos o fetales en los embarazos expuestos a glargina frente a NPH ${ }^{33}$. La insulina detemir demostró que no existen diferencias en su uso comparado con la NPH en los resultados maternos o neonatales. El control glucémico fue discretamente mejor con detemir en comparación con la NPH, así como menos riesgo de hipoglucemia materna y niveles de $\mathrm{HbA} 1 \mathrm{c}$ ligeramente menores ${ }^{34}$. La insulina basal puede suministrarse en dos dosis de NPH o con uno de los análogos de acción prolongada, siendo preferible detemir a glargina. No se han realizado estudios que analicen la seguridad de las 
Tabla 4. Uso de insulinas durante el embarazo según las diferentes agencias reguladoras

\begin{tabular}{llll}
\hline Nombre de la Insulina & Perfil de acción & Guía de la US FDA & Guía de la EMA \\
\hline Insulina regular & Acción corta & Categoría B* & Se puede utilizar \\
\hline Insulina NPH & Acción intermedia & Categoría B & Se puede utilizar \\
\hline Insulina lispro & Acción rápida & Categoría B & Se puede utilizar \\
\hline Insulina aspart & Acción rápida & Categoría B & Se puede utilizar \\
\hline Insulina glulisina & Acción rápida & Categoría C & Sin información clínica \\
\hline Insulina detemir & Acción prolongada & Categoría B & Se puede utilizar \\
\hline Insulina glargina & Acción prolongada & Sin información para embarazo en & Sin información clínica \\
\hline Insulina degludec & Acción ultralarga & Categoría C & Sin información clínica \\
\hline
\end{tabular}

*De acuerdo con la FDA: la categoría B hace referencia a que los estudios de reproducción animal de fármacos relacionados no han demostrado un riesgo para el feto y no hay estudios adecuados y bien controlados en mujeres embarazadas; la categoría C significa que los estudios de reproducción animal han mostrado un efecto adverso en el feto y no hay suficientes y estudios bien controlados en humanos, pero los beneficios potenciales pueden justificar el uso del fármaco en mujeres embarazadas a pesar de los riesgos potenciales. Sin embargo, la FDA descartó este sistema de letras en 2015 y lo reemplazó con un nuevo sistema.

US FDA: Administración de Alimentos y Medicamentos de los Estados Unidos; EMA: Agencia Europea de Medicamentos.

Adaptada de Jethwani, et al., $2021^{38}$.

insulinas basales más recientes como degludec, glargina U300 y el biosimilar de glargina. La insulina basal degludec parece ser segura en el embarazo, aunque no es la insulina que se prefiere; hay varias series de casos pequeños de mujeres sin resultados adversos observados ${ }^{35}$.

En algunos casos de DM2 y en todos los casos de DM1 se deberán utilizar insulinas de corta acción (asociadas a la insulina basal) como parte del control glucémico, idealmente bajo un esquema de conteo de carbohidratos, en vez de dosis fijas. Se prefiere el uso de insulina lispro o aspart en vez de la insulina regular, ya que su inicio de acción es más rápido, lo que permite un mejor control glucémico posprandial ${ }^{36}$, además de que existen mayores datos de seguridad. La satisfacción de los pacientes también ha sido mayor para los que utilizan lispro o aspart en comparación con la insulina regular. No existen datos sobre la insulina glulisina ni la insulina aspart de acción ultrarrápida (aprobada por la FDA) en el embarazo.

La insulina lispro o aspart pueden ser especialmente útiles en mujeres con hiperémesis o gastroparesia, ya que pueden dosificarse después de una comida y seguir siendo eficaces. Las insulinas de acción rápida pueden tardar más en alcanzar las concentraciones máximas (49 frente a $71 \mathrm{~min}$ ) al final del embarazo ${ }^{37}$. Por lo tanto, para algunas mujeres puede ser necesario administrar la insulina 15-30 minutos antes del comienzo del consumo de alimentos.
En la tabla 4 se enumeran las distintas insulinas aprobadas por las distintas agencias regulatorias ${ }^{38}$.

\section{USO DE INSULINA EN ADULTOS MAYORES}

Dos de los factores que con mayor frecuencia se asocian a una mayor prevalencia de diabetes son el peso corporal y la edad. Más de una cuarta parte de las personas mayores de 65 años tiene diabetes y la mitad de los adultos mayores prediabetes, según datos publicados en EE.UU. ${ }^{39}$. Es importante reconocer dos grupos de personas con diabetes mayores de 65 años: aquellos que llevan varios años con el diagnóstico y aquellos que son diagnosticados en esta edad. Los primeros tienen mayor probabilidad presentar complicaciones microvasculares o macrovasculares al llegar a esta edad. Los segundos corren el riesgo de desarrollar complicaciones microvasculares similar al de sus homólogos más jóvenes, aunque probablemente el riesgo absoluto sea menor si desarrollan la diabetes más tarde, lo que limitará su duración. Sin embargo, su riesgo absoluto de complicaciones macrovasculares es sustancialmente mayor que el de las personas más jóvenes con diabetes. En cualquiera de los dos grupos existe un mayor riesgo de tener polifarmacia, discapacidades funcionales y otros síndromes geriátricos 
Tabla 5. Consideraciones de la salud general y objetivos glucémicos del paciente adulto mayor con diabetes

\begin{tabular}{|c|c|c|c|c|}
\hline \multicolumn{2}{|l|}{$\begin{array}{l}\text { Categoría de salud } \\
\text { general }\end{array}$} & $\begin{array}{l}\text { Grupo 1: } \\
\text { buena salud }\end{array}$ & $\begin{array}{l}\text { Grupo 2: } \\
\text { salud intermedia }\end{array}$ & $\begin{array}{l}\text { Grupo 3: } \\
\text { mala salud }\end{array}$ \\
\hline \multirow{2}{*}{\multicolumn{2}{|c|}{ Características del paciente }} & $\begin{array}{l}\text { Sin comorbilidades } \\
0 \\
1-2 \text { enfermedades crónicas no } \\
\text { relacionadas a DM* } \\
\text { y } \\
\text { sin impedimentos para la } \\
\text { realización de AVD y } \leq 1 \\
\text { impedimento para la realización } \\
\text { de AIVD }\end{array}$ & $\begin{array}{l}\geq 3 \text { enfermedades crónicas no } \\
\text { relacionadas a } \mathrm{DM}^{*} \\
\text { y/o } \\
\text { cualquiera de los siguientes: } \\
\text { - Disfunción cognitiva } \\
\quad \text { moderada o demencia } \\
\text { temprana } \\
\text { - } \geq 2 \text { impedimentos para la } \\
\text { realización de AIVD }\end{array}$ & $\begin{array}{l}\text { Cualquiera de los siguientes: } \\
\text { - Condiciones médicas en etapa } \\
\text { terminal }{ }^{\dagger} \\
\text { - Demencia moderada a severa } \\
\text { - } \geq 2 \text { impedimentos para la } \\
\text { realización de AVD. } \\
\text { - Residencia a largo plazo en un } \\
\text { asilo }\end{array}$ \\
\hline & & Rangos razor & $\begin{array}{l}\text { nables de metas de glucosa y H } \\
\text { Toma de decisiones compartic } \\
\text { dividualizadas pueden ser men }\end{array}$ & c por grupo \\
\hline \multirow{2}{*}{\multicolumn{2}{|c|}{$\begin{array}{l}\text { Uso de medicamentos Nc } \\
\text { que puedan causar } \\
\text { hipoglucemia (p. ej., } \\
\text { insulina, sulfonilureas, } \\
\text { glinidas) }\end{array}$}} & $\begin{array}{l}\text { Ayuno: } 90-130 \mathrm{mg} / \mathrm{dl} \\
\text { Antes de ir a dormir: } \\
90-150 \mathrm{mg} / \mathrm{dl} \\
<7.5 \%\end{array}$ & $\begin{array}{l}\text { Ayuno: } 90-150 \mathrm{mg} / \mathrm{dl} \\
\text { Antes de ir a dormir: } \\
100-180 \mathrm{mg} / \mathrm{dl} \\
<8 \%\end{array}$ & $\begin{array}{l}\text { Ayuno: } 100-180 \mathrm{mg} / \mathrm{dl} \\
\text { Antes de ir a dormir: } \\
110-200 \mathrm{mg} / \mathrm{dl} \\
<8.5 \%\end{array}$ \\
\hline & & $\begin{array}{l}\text { Ayuno: } 90-150 \mathrm{mg} / \mathrm{dl} \\
\text { Antes de ir a dormir: } \\
100-180 \mathrm{mg} / \mathrm{dl} \\
\geq 7.0 \text { y }<7.5 \%\end{array}$ & $\begin{array}{l}\text { Ayuno: } 100-150 \mathrm{mg} / \mathrm{dl} \\
\text { Antes de ir a dormir: } \\
150-180 \mathrm{mg} / \mathrm{dl} \\
\geq 7.5 \mathrm{y}<8.0 \%\end{array}$ & $\begin{array}{l}\text { Ayuno: } 100-180 \mathrm{mg} / \mathrm{dl} \\
\text { Antes de ir a dormir: } \\
150-250 \mathrm{mg} / \mathrm{dl} \\
\geq 8.0 \mathrm{y}<8.5 \%{ }^{\S}\end{array}$ \\
\hline
\end{tabular}

* Las enfermedades crónicas coexistentes pueden incluir osteoartritis, hipertensión, enfermedad renal crónica en etapas 1-3 o accidente cerebrovascular, entre otras. †Una o más enfermedades crónicas con tratamientos limitados y esperanza de vida reducida. Estas incluyen cáncer metastásico, enfermedades pulmonares dependientes de oxígeno, enfermedad renal en etapa terminal que requiere diálisis e insuficiencia cardiaca avanzada.

‡Siempre y cuando puedan lograrse sin llegar a una hipoglucemia clínicamente significativa; de lo contrario, los objetivos de glucosa más altos pueden ser apropiados. Tenga en cuenta también que se incluyeron los límites inferiores de HbA1c, ya que los datos que sugieren un aumento de hipoglucemia y riesgo de mortalidad con niveles más bajos de HbA1c son más fuertes en el contexto del uso de insulina. Sin embargo, el límite inferior no debe reducir la vigilancia para una evaluación detallada de la hipoglucemia.

ङUna $\mathrm{HbA1c}$ del 8.5\% está correlacionada con un nivel de glucosa promedio de aproximadamente $200 \mathrm{mg} / \mathrm{dl}$. Metas más altas pueden resultar en glucosuria, deshidratación, crisis hiperglucémica y cicatrización de heridas deficiente.

'Las AVD incluyen bañarse, vestirse, alimentarse, ir al baño, trasladarse y las AIVD incluyen preparar alimentos, ir de compras, manejar dinero, usar el teléfono y manejar medicamentos.

DM: diabetes mellitus; AVD: actividades de la vida diaria; AIVD: actividades instrumentales de la vida diaria.

Adaptada de LeRoith, et al., $2019^{41}$.

comunes que incluyen el deterioro cognitivo, la depresión, la incontinencia urinaria, las caídas y el dolor persistente ${ }^{40}$. Es por ello importante que al momento de decidir el tratamiento antidiabético de las personas adultas mayores se prescriban esquemas diseñados en el estado de salud del paciente, sus comorbilidades y específicamente para disminuir el riesgo de hipoglucemia, ya que esta puede incrementar la morbimortalidad ${ }^{40}$. Como está recomendado por las distintas guías internacionales ${ }^{41-43}$, la metformina es el medicamento oral inicial para el control de la glucemia, sin dejar de enfatizar por los cambios del estilo de vida, que son muy relevantes. Si el paciente no alcanza el objetivo glucémico (el cual deberá ser establecido desde el inicio y dependerá de las características particulares de cada paciente) (Tabla 5) se pueden adicionar otros agentes orales o inyectables (idealmente con bajo riesgo de hipoglucemia), así como la insulina.

En la actualidad, la insulina suele iniciarse cuando los agentes orales y/o los AR-GLP-1 no proporcionan un control glucémico suficiente o no son asequibles $^{42}$. Las indicaciones del uso de insulina en los pacientes adultos mayores son similares que en personas de menor edad, sobre todo en aquellas personas que tengan hiperglucemia moderada/severa o persistente a pesar del uso de otras terapias. Puede considerarse un tratamiento inicial para los pacientes con diabetes de tipo 2, especialmente los que presentan una $\mathrm{HbA} 1 \mathrm{c}>9 \%$, glucosa plasmática en ayunas $>250 \mathrm{mg} / \mathrm{dl}$, una glucosa aleatoria 
constante $>300 \mathrm{mg} / \mathrm{dl}$ o cetonuria, o con valores de glucosa y $\mathrm{HbA} 1 \mathrm{c}$ menores en pacientes que ya tengan terapia oral/inyectable no insulínica. Debido a la preocupación por la hipoglucemia, el monitoreo de glucosa debe ser más frecuente (mediante glucómetros convencionales o monitoreo continuo de la glucosa), además de la $\mathrm{HbA} 1 \mathrm{c}$. Si se logra mejorar la secreción y/o la sensibilidad a la insulina (p. ej., en pacientes que presentaron alguna infección y desarrollaron hiperglucemia), la dosis puede reducirse o sustituirse por otras terapias con menor riesgo de hipoglucemia. En pacientes de 65 años o más con diabetes que reciben tratamiento con insulina, se recomienda un monitoreo de glucosa más frecuente de la glucosa.

Antes de iniciar el tratamiento con insulina en un paciente adulto mayor, es importante evaluar si el paciente es capaz, tanto física como cognitivamente, de utilizar una pluma de insulina o de extraer y administrar la dosis adecuada de insulina, de monitorear la glucemia y de reconocer/tratar la hipoglucemia. Aunque es recomendable que los pacientes se apliquen la insulina por sí mismos para conservar su independencia, en algunos casos puede ser complejo para el paciente, por lo que será necesario que un familiar/cuidador sea quien administre el tratamiento, teniendo en mente que debe monitorear la glucemia y buscar intencionadamente datos de hipoglucemia ${ }^{43}$.

En el caso de los pacientes con fragilidad, el tratamiento hipoglucemiante puede ser más complejo, y la falta de guías locales o internacionales, sobre todo en pacientes que tienen múltiples comorbilidades, son factores que pueden generar inercia clínica. En algunos pacientes es necesario valorar/ eliminar algunas prescripciones o desescalar tratamiento que pueda generar más daño que beneficio. Debemos identificar y evaluar las complicaciones y comorbilidades avanzadas de los adultos mayores con DM2, los aspectos médicos, psicológicos y funcionales, como la sarcopenia, insuficiencia cardiaca, función renal, hipertensión, perfil lipídico, incontinencia urinaria, deterioro cognitivo, depresión, dieta, estado físico, caídas y fracturas, dificultad para la deglución, apoyo de terceras personas para la administración de los medicamentos, así como la polifarmacia, que puedan interferir con la adherencia y la respuesta farmacodinámica a los tratamien$\operatorname{tos}^{43}$.

En algunas ocasiones (y debido a factores que tienen que ver con el médico, el paciente o la familia) la insulina se infrautiliza en los adultos mayores por temor a que sea complejo o peligroso. Para evitar esto, se sugiere esquemas más simples. Por ejemplo, puede agregarse una insulina basal de larga duración (idealmente análoga por el menor riesgo de hipoglucemia) a una terapia no insulínica (metformina \pm otra terapia oral con bajo riesgo de hipoglucemia) y titularse hasta lograr los objetivos glucémicos personalizados, con lo que se logra un esquema de baja complejidad, con un riesgo menor de hipoglucemia, en comparación con los esquemas que utilizan múltiples dosis de insulina. La dosis de la insulina de acción prolongada puede ajustarse una vez por semana y lentamente para evitar hipoglucemias, hasta alcanzar el objetivo de glucemia ${ }^{42}$. La insulina glargina U300 y la insulina degludec han mostrado niveles de control glucémico similares, pero con menos variabilidad glucémica e hipoglucemia ${ }^{41}$ comparadas contra otras insulinas basales.

Si los pacientes presentan excursiones de glucosa posprandial, sobre todo en aquellos con glucosas de ayuno adecuadas pero con HbA1c elevada, se debe intensificar el tratamiento. Entre las opciones disponibles en la actualidad, las combinaciones de proporción fija con AR-GLP-1 e insulina basal, por ejemplo insulina degludec y liraglutida e insulina glargina y lixisenatida, están ganando particular atención, ya que se encuentran disponibles para una sola aplicación en un dispositivo. Los estudios han informado de una adecuada reducción de la $\mathrm{HbA1c}$ con menos hipoglucemia y menor impacto sobre el aumento de peso ${ }^{44}$. Otra opción de intensificación es añadir a la insulina basal una insulina de acción rápida, antes de la comida más importante y luego antes de otras comidas, según sea necesario. También las insulinas premezcladas administradas dos veces al día pueden ser eficaces para intensificar, pero con menor flexibilidad, especialmente en los pacientes que se saltan o retrasan los alimentos, lo que puede aumentar el riesgo de hipoglucemia. Lo ideal es evitar al máximo esquemas complejos, ya que puede tener impacto en la adherencia y el riesgo de hipoglucemia. 


\section{USO DE INSULINA EN ENFERMEDAD} RENAL CRÓNICA

Las complicaciones microvasculares derivadas de la diabetes han determinado en los últimos años el destino de las personas con esta enfermedad. Conforme la mejoría de la expectativa de vida de la población, una mejor prevención cardiovascular, así como una mayor duración de la diabetes gracias a mejores terapias y al cuidado del paciente con diabetes, nuestro enfoque en los siguientes años debería de enfatizarse hacia el control de las complicaciones microvasculares ${ }^{45}$. Desafortunadamente un porcentaje importante de las personas con diabetes no alcanzan objetivos glucémicos adecuados por largos periodos de tiempo. Gracias a los importantes estudios clásicos de la diabetología, sabemos que la $\mathrm{HbA1c}$ es un factor fuerte e independiente de la enfermedad microvascular. En el estudio UK Prospective Diabetes Study (UKPDS), la intensificación del tratamiento con insulina tuvo un efecto significativo en la enfermedad de los microvasos, con una estrecha relación entre la $\mathrm{HbA} 1 \mathrm{c}$ y el descenso de la retinopatía a $\mathrm{HbA} 1 \mathrm{c}<6 \%$ tras 10 años de seguimiento ${ }^{46}$. En este mismo estudio, la adición temprana de insulina al tratamiento oral redujo el riesgo de complicaciones. Otro de los estudios que demostró que la adición temprana y oportuna con insulina permitía una reducción significativa de la enfermedad renal crónica (ERC) y la retinopatía fue el ORIGIN, sobre todo en aquellos participantes con una $\mathrm{HbA} 1 \mathrm{c}$ basal $>6.4 \%{ }^{47}$.

Por otro lado, una vez que ya se encuentra establecida la ERC, es necesario tomar en cuenta que el tratamiento hipoglucemiante debe modificarse de acuerdo con la tasa de filtrado glomerular estimada del paciente (TFGe) y otros factores individuales. La ERC se asocia a Rl y el control glucémico puede deteriorarse a medida que la función renal disminuye. La sensibilidad tisular a la insulina está alterada en casi todas las personas urémicas y es en gran medida responsable del metabolismo anormal de la glucosa que se observa en este contexto. Los posibles mecanismos que podrían explicar la reducción del manejo de la glucosa mediado por la insulina incluyen: 1) un aumento de la gluconeogénesis hepática que no se suprime normalmente tras la administración de insulina;
2) una reducción de la captación de glucosa hepática y/o del músculo esquelético, y 3 ) un deterioro del metabolismo intracelular de la glucosa debido a una menor oxidación a dióxido de carbono y agua o a una menor síntesis de glucógeno. Sin embargo, el músculo esquelético es el lugar principal de la RI. Aunque se desconoce el mecanismo exacto, se cree que la acumulación de las toxinas urémicas y el exceso de hormona paratiroidea son responsables de la RI. La mejoría en la sensibilidad a la insulina que ocurre después de la diálisis coincide con estas observaciones ${ }^{48}$.

Cuando la ERC es avanzada, se produce una reducción importante de la depuración de la insulina, lo que lleva a una disminución de las necesidades de insulina o incluso al cese del tratamiento en pacientes con DM2. En las etapas iniciales de las ERC, la tasa de eliminación metabólica de la insulina apenas cambia, hasta que se produce una reducción sustancial de la TFG. El aumento en la captación peritubular de la insulina es capaz de compensar la reducción de la filtración, hasta que la TFG ha descendido a menos de 15 a $20 \mathrm{~mL} / \mathrm{min}$. En este punto se produce una reducción drástica de la depuración de insulina, que también está mediada por una disminución concomitante del metabolismo hepático de la insulina ${ }^{49}$.

Con estos dos procesos identificados en los pacientes con diabetes y ERC se puede observar que las necesidades de insulina en estos pacientes muestran dos etapas. En la primera etapa no es infrecuente que el control de la glucosa se deteriore a medida que se deteriora la función renal y aumenta la RI. Por lo tanto, la instauración de insulina o aumento en las necesidades de insulina pueden ser necesarias. En la segunda etapa, con mayor deterioro de la función renal, es probable sea necesario una menor dosis de insulina o incluso su suspensión. En ocasiones, la disminución de la ingesta calórica debida a la hiporexia inducida por la uremia también puede contribuir a la disminución de las necesidades de insulina ${ }^{48-50}$.

Las terapias hipoglucemiantes en pacientes con deterioro de la función renal pueden ser fármacos orales, inyectables no insulínicos e insulina. Sin embargo, con una TFG por debajo de $30 \mathrm{~mL} / \mathrm{min} / 1.73 \mathrm{~m}^{2}$, pero sobre todo $<15 \mathrm{~mL} / \mathrm{min} / 1.73 \mathrm{~m}^{2}$ un gran número de las terapias hipoglucemiantes están contraindicadas, por lo que solo pocos fármacos orales 
y la insulina podrían utilizarse en este grupo de pacientes.

Debido al mayor riesgo de hipoglucemia asociado a la ERC, la insulina debe utilizarse con precaución, sobre todo en pacientes con deterioro avanzado de la TFG. Por otro lado, los distintos estudios han demostrado que las insulinas (tanto humanas como análogas) son seguras en todas las categorías de ERC. Sobre todo las insulinas humanas (debido a que se metabolizan por la insulinasa del hígado y del riñón) deberán de reducirse en sus dosis prescritas. La dosis inicial de insulina puede tener que ser inferior a la que se utilizaría normalmente en pacientes con una función renal normal. Se puede realizar un ajuste a la dosis de insulina que vaya acorde al deterioro de la función renal ${ }^{50}$.

- TFGe > $50 \mathrm{~mL} / \mathrm{min} / 1.73 \mathrm{~m}^{2}$ : no se necesita ajuste.

- TFGe entre 10-50 mL/min/1.73 $\mathrm{m}^{2}$ : disminuir al $75 \%$ de la dosis basal.

- TFGe $<10 \mathrm{~mL} / \mathrm{min} / 1.73 \mathrm{~m}^{2}$ : disminuir al 50\% de la dosis basal.

\section{AGRADECIMIENTOS}

Los autores agradecen a la mesa directiva 2021 de la Sociedad Mexicana de Nutrición y Endocrinología, así como al coordinador general del suplemento, por la invitación para escribir este artículo.

\section{FINANCIAMIENTO}

La presente investigación no ha recibido ninguna beca específica de agencias de los sectores públicos, comercial o con ánimo de lucro.

\section{CONFLICTO DE INTERESES}

Los autores declaran no tener conflicto de intereses.

\section{RESPONSABILIDADES ÉTICAS}

Protección de personas y animales. Los autores declaran que para esta investigación no se han realizado experimentos en seres humanos ni en animales.

Confidencialidad de los datos. Los autores declaran que en este artículo no aparecen datos de pacientes.

Derecho a la privacidad y consentimiento informado. Los autores declaran que en este artículo no aparecen datos de pacientes.

\section{BIBLIOGRAFÍA}

1. American Diabetes Association. 9. Pharmacologic Approaches to Glycemic Treatment: Standards of Medical Care in Diabetes-2021. Diabetes Care. 2021:44(Suppl.1):S111-S124.

2. Davies MJ, D'Alessio DA, Fradkin J, Kernan WN, Mathieu C, Mingrone G, et al. Management of Hyperglycemia in Type 2 Diabetes, 2018. A Consensus Report by the American Diabetes Association (ADA) and the European Association for the Study of Diabetes (EASD). Diabetes Care. 2018;41(12):2669-701.

3. Diabetes Canada Clinical Practice Guidelines Expert Committee Lipscombe L, Butalia S, Dasgupta K, Eurich DT, MacCallum L, Shah BR, et al. Pharmacologic glycemic management of type 2 diabetes in adults: 2020 update. Can J Diabetes. 2020;44(7):575-91.

4. Guías ALAD sobre el Diagnóstico, Control y Tratamiento de la Diabetes Mellitus Tipo 2 con Medicina Basada en Evidencia. Edición 2019 [Internet]. Asociación Latinoamericana de Diabetes; 2019. Disponible en https://www.revistaalad.com/guias/5600AX191 guias alad_2019.pdf

5. Accili D. Lilly lecture 2003: the struggle for mastery in insulin action: from triumvirate to republic. Diabetes. 2004;53(7):1633-42.

6. Malakar P, Chartarifsky L, Hija A, Leibowitz G, Glaser B, Dor Y, et al. Insulin receptor alternative splicing is regulated by insulin signaling and modulates beta cell survival. Sci Rep. 2016;6:31222.

7. Beale EG. Insulin signaling and insulin resistance. J Invest Med. 2013;61(1):11-4.

8. Kahn CR, Rosenthal AS. Immunologic reactions to insulin: insulin allergy, insulin resistance, and the autoimmune insulin syndrome. Diabetes Care. 1979;2(3):283-95.

9. Petersen KF, Dufour S, Savage DB, Bilz S, Solomon G, Yonemitsu S, et al. The role of skeletal muscle insulin resistance in the pathogenesis of the metabolic syndrome. Proc Natl Acad Sci U S A. 2007;104(31):12587-94.

10. Angelidi AM, Filippaios A, Mantzoros CS. Severe insulin resistance syndromes. J Clin Invest. 2021;131(4):e142245.

11. Cruz PD Jr, Hud JA Jr. Excess insulin binding to insulin-like growth factor receptors: proposed mechanism for acanthosis nigricans. J Invest Dermatol. 1992;98(6 Suppl I.):82S-85S.

12. Melvin A, O'Rahilly S, Savage DB. Genetic syndromes of severe insulin resistance. Curr Opin Genet Dev. 2018;50:60-7.

13. Buchanan TA, Watanabe RM, Xiang AH. Limitations in surrogate measures of insulin resistance. J Clin Endocrinol Metab. 2010;95(11):4874-6.

14. Umpierrez GE, Holt EH, Einhorn D, McGill JB. Concentrated insulins: clinical update of therapeutic options. Endocr Pract. 2020;26(Suppl 3):1-12.

15. de la Peña A, Seger M, Soon D, Scott AJ, Reddy SR, Dobbins MA, et al. Bioequivalence and comparative pharmacodynamics of insulin lispro $200 \mathrm{U} / \mathrm{mL}$ relative to insulin lispro (Humalog ${ }^{\circledast}$ ) $100 \mathrm{U} / \mathrm{mL}$. Clin Pharmacol Drug Dev. 2016;5(1):69-75.

16. Gough SC, Bhargava A, Jain R, Mersebach $H$, Rasmussen $S$, Bergenstal RM. Low-volume insulin degludec 200 units $/ \mathrm{mL}$ once daily improves glycemic control similarly to insulin glargine with a low risk of hypo- 
glycemia in insulin-naive patients with type 2 diabetes: a 26-week, randomized, controlled, multinational, treat-to-target trial: the BEGIN LOW VOLUME trial. Diabetes Care. 2013;36(9):2536-42.

17. Hurren KM, O'Neill JL. Pharmacodynamic and pharmacokinetic evaluation of insulin glargine U300 for the treatment of type 1 diabetes. Expert Opin Drug Metab Toxicol. 2016;12(12):1521-6.

18. Ritzel R, Roussel R, Bolli GB, Vinet $L$, Brulle-Wohlhueter C, Glezer $S$, et al. Patient-level meta-analysis of the EDITION 1, 2 and 3 studies: glycaemic control and hypoglycaemia with new insulin glargine $300 \mathrm{U} / \mathrm{mL}$ versus glargine $100 \mathrm{U} / \mathrm{mL}$ in people with type 2 diabetes. Diabetes Obes Metab. 2015;17(9):859-67.

19. Mikhail N. Insulin U-500, the Practical Solution for the treatment of patients with high insulin requirements. Curr Diabetes Rev. 2021;17(1): 26-9.

20. Shrestha RT, Kumar AF, Taddese A, Khowaja AA, Moheet A, Olawsky E, et al. Duration and onset of action of high dose $U-500$ regular insulin in severely insulin resistant subjects with type 2 diabetes. Endocrinol Diabetes Metab. 2018;1(4):e00041.

21. Kohli R, Shevitz A, Gorbach S, Wanke C. A randomized placebo-controlled trial of metformin for the treatment of HIV lipodystrophy. HIV Med. 2007;8(7):420-6.

22. Agostini M, Schoenmakers E, Beig J, Fairall L. A pharmacogenetic approach to the treatment of patients with PPARG mutations. diabetes. 2018;67(6):1086-92.

23. GBD 2015 Obesity Collaborators, Afshin A, Forouzanfar MH, Reitsma MB, Sur P, Estep K, Lee A, et al. Health effects of overweight and obesity in 195 countries over 25 years. N Engl J Med. 2017;377(1):13-27.

24. Barquera S, Hernández-Barrera L, Trejo-Valdivia B, Shamah T, CamposNonato I, Rivera-Dommarco J. Obesidad en México, prevalencia y tendencias en adultos. Ensanut 2018-19. Salud Publ Mex. 2020;62(6): 682-92.

25. GBD 2016 DALYs and HALE Collaborators. Global, regional, and national disability-adjusted life-years (DALYs) for 333 diseases and injuries and healthy life expectancy (HALE) for 195 countries and territories, 19902016: a systematic analysis for the Global Burden of Disease Study 2016. Lancet. 2017;390(10100):1260-344.

26. Church TJ, Haines ST. Treatment approach to patients with severe insulin resistance. Clin Diabetes. 2016;34(2):97-104.

27. Buschur EO, Polsky S. Type 1 diabetes: Management in women from preconception to postpartum. J Clin Endocrinol Metab. 2021;106(4): 952-67.

28. Retnakaran R. Diabetes in pregnancy 100 years after the discovery of insulin: Hot topics and open questions to be addressed in the coming years. Metabolism. 2021;119:154772.

29. Ludvigsson JF, Neovius M, Söderling J, Gudbjörnsdottir S, Svensson AM, Franzén $S$, et al. Periconception glycaemic control in women with type 1 diabetes and risk of major birth defects: population based cohort study in Sweden. BMJ. 2018;362:k2638.

30. International Diabetes Federation. IDF Diabetes Atlas, 9th ed. Bruselas, Bélgica: International Diabetes Federation; 2019. Disponible en: https:// www.diabetesatlas.org

31. American Diabetes Association. 2. Classification and Diagnosis of Diabetes: Standards of Medical Care in Diabetes-2021. Diabetes Care. 2021;44(Suppl.1):S15-S33.

32. ACOG Practice Bulletin No. 190 Summary: Gestational diabetes mellitus. Obstet Gynecol. 2018;131(2):406-8.

33. Lepercq J, Lin J, Hall GC, Wang E, Dain MP, Riddle MC, et al. Metaanalysis of maternal and neonatal outcomes associated with the use of insulin glargine versus NPH insulin during pregnancy. Obstet Gynecol Int. 2012;2012:649070.

34. Mathiesen ER, Hod M, Ivanisevic M, Duran Garcia S, Brøndsted L, Jovanovic $L$, et al.; Detemir in Pregnancy Study Group. Maternal efficacy and safety outcomes in a randomized, controlled trial comparing insulin detemir with NPH insulin in 310 pregnant women with type 1 diabetes. Diabetes Care. 2012;35(10):2012-7.

35. Hiranput S, Ahmed SH, Macaulay D, Azmi S. Successful outcomes with insulin degludec in pregnancy: A case series. Diabetes Ther. 2019;10(1): 283-9.

36. Hod M, Damm P, Kaaja R, Visser GH, Dunne F, Demidova I, et al.; Insulin Aspart Pregnancy Study Group. Fetal and perinatal outcomes in type 1 diabetes pregnancy: a randomized study comparing insulin aspart with human insulin in 322 subjects. Am J Obstet Gynecol. 2008;198(2): 186.e1-7.

37. Murphy HR, Elleri D, Allen JM, Harris J, Simmons D, Rayman G, et al. Pathophysiology of postprandial hyperglycaemia in women with type 1 diabetes during pregnancy. Diabetologia. 2012;55(2):282-93.

38. Jethwani P, Saboo B, Jethwani L, Chawla R, Maheshwari A, Agarwal S, et al. Use of insulin glargine during pregnancy: A review. Diabetes Metab Syndr. 2021;15(1):379-84.

39. Centers for Disease Control and Prevention. National Diabetes Statistics Report: estimates of diabetes and its burden in the United States, 2020 [Internet]. EE.UU.: Centers for Disease Control and Prevention; 2020. Disponible en: https://www.cdc.gov/diabetes/pdfs/data/statistics/national-diabetes-statistics-report.pdf

40. Kirkman MS, Briscoe VJ, Clark N, Florez H, Haas LB, Halter JB, et al.; Consensus Development Conference on Diabetes and Older Adults. Diabe tes in older adults: a consensus report. J Am Geriatr Soc. 2012;60(12): 2342-56.

41. LeRoith D, Biessels GJ, Braithwaite SS, Casanueva FF, Draznin B, Halter JB, et al. Treatment of diabetes in older adults: An Endocrine Society Clinical Practice Guideline. J Clin Endocrinol Metab. 2019;104(5): 1520-74.

42. American Diabetes Association. 12. Older Adults: Standards of Medical Care in Diabetes-2021. Diabetes Care. 2021;44(Suppl 1):S168-S179.

43. Strain WD, Down S, Brown P, Puttanna A, Sinclair A. Diabetes and frailty: An expert consensus statement on the management of older adults with type 2 diabetes. Diabetes Ther. 2021;12(5):1227-47.

44. Maiorino Ml, Chiodini P, Bellastella G, Capuano A, Esposito K, Giugliano $D$. Insulin and glucagon-like peptide 1 receptor agonist combination therapy in type 2 diabetes: A systematic review and meta-analysis of randomized controlled trials. Diabetes Care. 2017;40(4):614-24.

45. Hanefeld M, Fleischmann H, Siegmund T, Seufert J. Rationale for timely insulin therapy in type 2 diabetes within the framework of individualised treatment: 2020 update. Diabetes Ther. 2020;11(8):1645-66.

46. Intensive blood-glucose control with sulphonylureas or insulin compared with conventional treatment and risk of complications in patients with type 2 diabetes (UKPDS 33). UK Prospective Diabetes Study (UKPDS) Group. Lancet. 1998;352(9131):837-53.

47. ORIGIN trial investigators, Gilbert RE, Mann JF, Hanefeld M, Spinas G, Bosch J, Yusuf S, et al. Basal insulin glargine and microvascular outcomes in dysglycaemic individuals: results of the Outcome Reduction with an Initial Glargine Intervention (ORIGIN) trial. Diabetologia. 2014;57(7): 1325-31.

48. Pugliese G, Penno G, Natali A, Barutta F, Di Paolo S, Reboldi G, et al.; Italian Diabetes Society and the Italian Society of Nephrology. Diabetic kidney disease: new clinical and therapeutic issues. Joint position statement of the Italian Diabetes Society and the Italian Society of Nephrology on "The natural history of diabetic kidney disease and treatment of hyperglycemia in patients with type 2 diabetes and impaired renal function". J Nephrol. 2020;33(1):9-35.

49. de Boer IH, Zelnick L, Afkarian M, Ayers E, Curtin L, Himmelfarb J, et al. Impaired glucose and insulin homeostasis in moderate-severe CKD. J Am Soc Nephrol. 2016;27(9):2861-71.

50. Snyder RW, Berns JS. Use of insulin and oral hypoglycemic medications in patients with diabetes mellitus and advanced kidney disease. Semin Dial. 2004;17(5):365-70. 